Latest Advances in Atomic Cluster Collisions

Structure and Dynamics from the Nuclear to the Biological Scale 
This page intentionally left blank 


\title{
Latest Advances in Atomic Cluster Collisions
}

Structure and Dynamics from the Nuclear to the Biological Scale

\author{
editors \\ Jean-Patrick Connerade \\ The Blackett Laboratory, Imperial College London, UK

\section{Andrey Solov'yov}

Frankfurt Institute for Advanced Studies,

Johann Wolfgang Goethe University, Germany 
Published by

Imperial College Press

57 Shelton Street

Covent Garden

London WC2H 9HE

Distributed by

World Scientific Publishing Co. Pte. Ltd.

5 Toh Tuck Link, Singapore 596224

USA office: 27 Warren Street, Suite 401-402, Hackensack, NJ 07601

UK office: 57 Shelton Street, Covent Garden, London WC2H 9HE

\section{British Library Cataloguing-in-Publication Data}

A catalogue record for this book is available from the British Library.

\section{LATEST ADVANCES IN ATOMIC CLUSTER COLLISIONS Structure and Dynamics from the Nuclear to the Biological Scale}

Copyright () 2008 by Imperial College Press

All rights reserved. This book, or parts thereof, may not be reproduced in any form or by any means, electronic or mechanical, including photocopying, recording or any information storage and retrieval system now known or to be invented, without written permission from the Publisher.

For photocopying of material in this volume, please pay a copying fee through the Copyright Clearance Center, Inc., 222 Rosewood Drive, Danvers, MA 01923, USA. In this case permission to photocopy is not required from the publisher.

ISBN-13 978-1-84816-237-2

ISBN-10 1-84816-237-5

Typeset by Stallion Press

Email: enquiries@ stallionpress.com

Printed in Singapore. 


\section{PREFACE}

The Second International Symposium "Atomic Cluster Collisions: Structure and dynamics from the nuclear to the biological scale" (ISACC 2007) was organized as a satellite meeting of the XXVth International Conference on Photonic, Electronic, and Atomic Collisions (ICPEAC 2007, Freiburg, Germany) by the Frankfurt Institute for Advanced Studies (FIAS) and Gesellschaft für Schwerionenforschung (GSI) and was held on July 19-23, 2007 at GSI, Darmstadt, Germany. ISACC was recognized by the European Physical Society (EPS) as a Europhysics Conference.

ISACC started as the international symposium on atomic cluster collisions in St. Petersburg, Russia in 2003. ISACC 2007 promoted significantly the growth and exchange of scientific information on the structure and properties of nuclear, atomic, molecular, biological and complex cluster systems studied by means of photonic, electronic, heavy particle and atomic collisions. In the symposium, particular attention was devoted to dynamical phenomena, many-body effects taking place in the clusters, molecular and biological systems, which include problems of fusion and fission, fragmentation, collective electron excitations, phase transitions and many more. Both experimental and theoretical aspects of cluster physics, uniquely placed between nuclear physics on one hand and atomic, molecular, condensed matter and solid state physics on the other, were the subject of the symposium.

The venue of the symposium was the Gesellschaft für Schwerionenforschung (GSI) at Darmstadt, which is one of the world-renowned research centers in the field of heavy ion physics. Several superheavy elements were synthesized here for the first time. This was a very natural location for a symposium on cluster science, since cluster properties of the nuclei are very thoroughly investigated and often appear as a prototype for similar phenomena in other many-body systems of various degrees of complexity.

The Symposium brought together more than 120 leading scientists in the field of atomic cluster physics from around the world. The special emphasis of the Symposium was on the new methods of investigation of the structure and properties of atomic clusters, the collective excitations 
in photoabsorption and photoionization processes of atomic clusters, fission and fusion dynamics of clusters, cluster dynamics in the laser field, resonance processes in electron-cluster collisions, the interaction of ions, including multiply charged ions, with metal clusters and fullerenes and the processes of cluster deposition on a surface as well as of cluster collisions on a surface. The aim of the Symposium was to present the most recent achievements in all these fields of atomic cluster science. These proceedings, we hope, bear witness that this goal has been fulfilled.

Part A of this book describes clustering phenomena at nuclear and subnuclear scales. It surveys recent advances in the synthesis of superheavy elements, clustering phenomena in fission and fusion processes of heavy nuclei and the properties of heavy and superheavy nuclei in supernova experiments.

Part $\mathrm{B}$ is devoted to recent advances in the understanding of structure and essential properties, such as nanomagnetism, electronic and geometric shell effects, of selected atomic cluster systems and confined atoms. Both theoretical and experimental aspects of the field are discussed.

Part $\mathrm{C}$ describes recent advances in electron, photon and ion cluster collisions. These include the problem of molecular rotation in external fields, electron scattering on neon droplets, dynamical screening of atoms by fullerene cages, photoionization and fragmentation of fullerene ions, and collisions of molecules with cluster ions.

Part D is devoted to the problem of clusters on a surface. In particular, it describes the recent efforts in infrared spectroscopy and thermal desorption of clusters on self-assembled monolayers and the atomistic approach to the simulation of the nanoindentation process.

Part E deals with the problems of phase transitions, fission, fusion and fragmentation in finite systems. In focus are quantum structuring of ${ }^{4} \mathrm{H}$ atoms around ionic dopants, phase transitions in polypeptides, and dissociation of charged rare gas clusters.

Part $\mathrm{F}$ of the book contains a discussion of clusters in laser fields. These include the dynamics of metal clusters in laser fields: phase, amplitude and polarization shaping by interferometric pulse generation.

Part $G$ reports on recent advances in the understanding of clustering phenomenon in systems of various degrees of complexity. This chapter includes the description of structure and stability of novel objects: electronpositron quantum droplets. The new data on spectroscopy of chromophores 
in the gas phase is covered, and energy landscape analysis as a computational tool for analysis of complex molecular systems is discussed.

Part $\mathrm{H}$ reports on recent advances in the understanding of structure and dynamics of biomolecules. These include a novel theoretical framework for the interpretation of NMR recidular dipolar couplings of unfolded proteins, computational simulation of antibody:antigen unbinding, mechanisms of magnetoreception in birds and biophysical modeling of fragment distributions of DNA plasmids after heavy ion irradiation.

Part I includes the discussion of several important biological problems manifesting themselves at the mesoscopic scale, in which dynamics, clustering and other properties of matter at smaller scales play the essential role. Case studies presented in this chapter include molecular and nuclear mechanisms of ion cancer therapy and analysis of gene expression patterns in the Drosophila embryo.

The subjects of the chapters in this book correspond to the sessions in the Symposium.

The organizers of the ISACC 2007 wish to acknowledge generous support received from the European Physical Society, the Frankfurt Institute for Advanced Studies, the Gesellschaft für Schwerionenforschung (GSI, Darmstadt), the European Commission within the Network of Excellence project EXCELL, which made this Symposium possible and a great success. The editors of this book would like to express their gratitude to Dr Andrey Lyalin, Dr Andriy Kostyuk, Dr Ilia Solov'yov, Mr Alexander Yakubovich and especially to Ms Stephanie Lo, for their great help in the preparation of the manuscript of this book for publication. Finally, we acknowledge our fruitful collaboration with Imperial College Press and the World Scientific Publishing Co.

Jean-Patrick Connerade

Imperial College, London, UK

Andrey V. Solov'yov

Frankfurt Institute for Advanced Studies, Germany

Chairman

The Second International Symposium "Atomic Cluster Collisions: Structure and dynamics from the nuclear to the biological scale"

Frankfurt am Main and London, 2007 
This page intentionally left blank 


\section{LOCAL ORGANISING COMMITTEE}
A. V. Solov'yov (Chairman) - FIAS, Germany
W. Greiner (Vice-Chair) - J.-W. Goethe University, Germany
S. Hofmann (Vice-Chair) — GSI, Germany
T. Haberer
- GSI, Germany
H. Feldmeier
- GSI, Germany
C. Fournier
- GSI, Germany
T. Litvinova
- GSI, Germany
A. G. Lyalin
- FIAS, Germany
O. I. Obolensky
- FIAS, Germany
S. Raiss
- GSI, Germany
I. A. Solov'yov
- FIAS, Germany
T. Stöhlker
- GSI, Germany

\section{INTERNATIONAL ADVISORY COMMITTEE}
U. Becker
- Fritz Haber Institute, Germany
C. Bréchignac
- CNRS, France
M. Broyer
- LASIM, University Lyon 1, France
E. Campbell
- Göteborg University, Sweden
A. W. Castleman Jr. - Pennsylvania State University, USA
J.-P. Connerade
- Imperial College London, UK
F. Gianturco
- The University of Rome "La Sapienza", Italy
H. Haberland
- University of Freiburg, Germany
B. Huber
- CIRIL, France
V. K. Ivanov
- St. Petersburg State Polytechnic University, Russia
J. Jellinek
- Argonne National Laboratory, USA
T. Kondow
- Toyota Technological Institute, Japan
B. Zhang
- Wuhan Institute of Physics and Mathematics, China 
This page intentionally left blank 


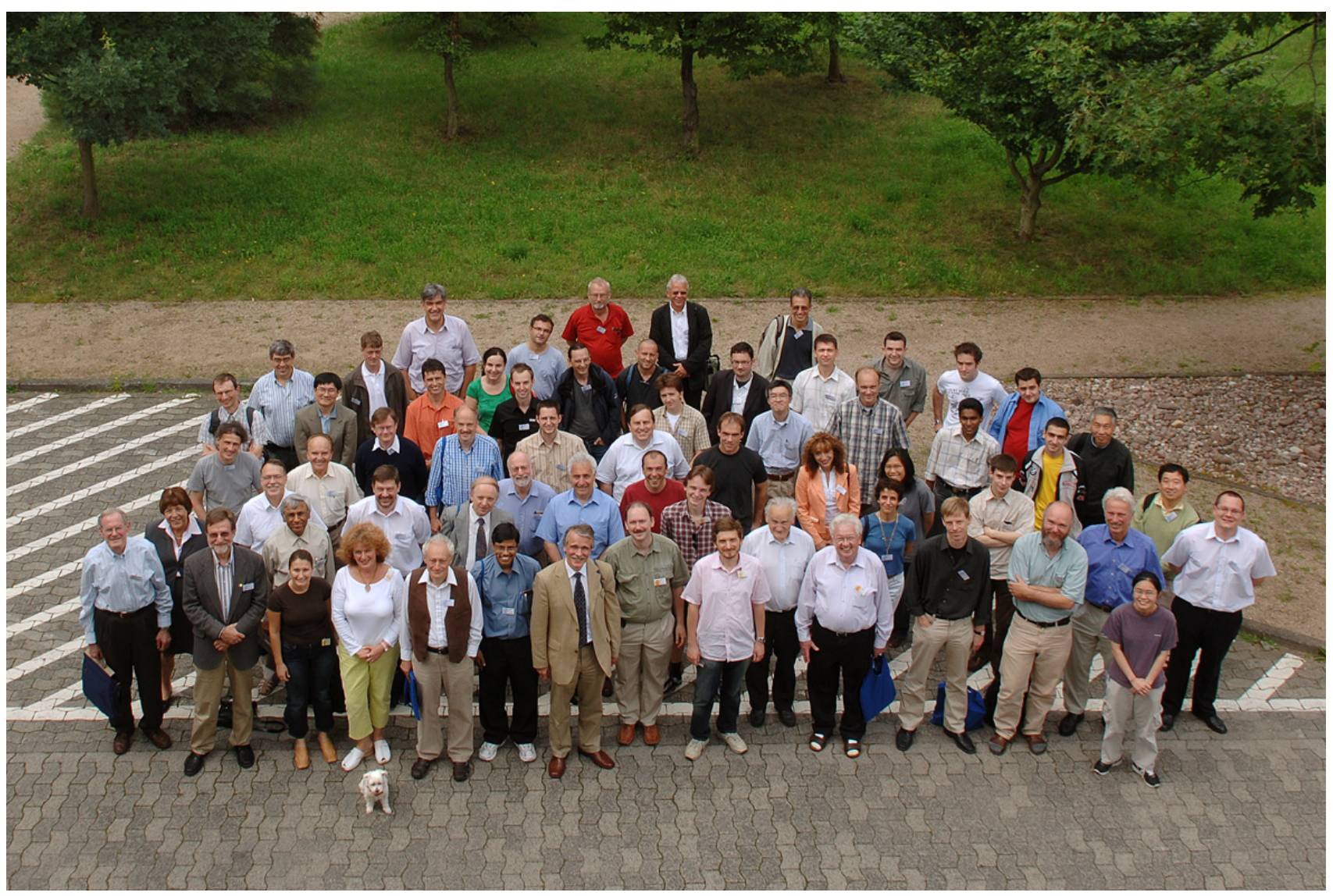


This page intentionally left blank 


\section{CONTENTS}

Preface $\quad$ V

Organizing Committees $\quad$ ix

Conference Photo $\quad$ xi

Part A. Clustering Phenomena at Nuclear

$\begin{array}{ll}\text { and Subnuclear Scales } & 1\end{array}$

Heaviest Nuclei from ${ }^{48}$ Ca-Induced Reactions $\quad 3$

Yu. Oganessian

Production and Decay of Superheavy Nuclei

S. Hofmann

Clustering Phenomena in Fission and Fusion Processes of Heavy Nuclei

V. Zagrebaev and W. Greiner

Nuclear Molecules

G. G. Adamian, A. V. Andreev, N. V. Antonenko,

S. P. Ivanova, R. V. Jolos, A. K. Nasirov,

T. M. Shneidman, A. S. Zubov and W. Scheid

Properties of Heavy and Superheavy Nuclei in Supernova Environments

T. J. Bürvenich, I. N. Mishustin and W. Greiner

Part B. Structure and Properties of Atomic Clusters

Clusters, Quantum Confinement and Energy Storage 
Site-Specific Analysis of Response Properties of Sodium Clusters

K. Jackson, M. Yang and J. Jellinek

Magnetism in Clusters

A. Lyalin, A. V. Solov'yov and W. Greiner

Strontium Clusters: Electronic and Geometry Shell Effects

A. Lyalin, I. A. Solov'yov, A. V. Solov'yov

and W. Greiner

New Deformed Single-Particle Shell Model

D. N. Poenaru, R. A. Gherghescu, I. H. Plonski,

A. V. Solov'yov and W. Greiner

\section{Part C. Electron, Photon and Ion Cluster Collisions}

Top Rotors in Electric Fields: Influence of the Asymmetry, the Flexibility and the Structure of the Molecules

M. Abd el Rahim, R. Antoine, M. Broyer,

P. Dugourd and D. Rayane

Electron Scattering on Neon Droplets: Singly and Multiply Charged Neon Clusters

S. Denifl, F. Zappa, I. Mähr, P. Scheier,

O. Echt and T. D. Märk

Dynamical Screening of an Atom Confined Within

a Finite-Width Fullerene

S. Lo, A. V. Korol and A. V. Solov'yov

Photoionization and Fragmentation of Fullerene Ions

A. Müller, S. Schippers, R. A. Phaneuf, S. Scully,

E. D. Emmons, M. F. Gharaibeh, M. Habibi,

A. L. D. Kilcoyne, A. Aguilar, A. S. Schlachter,

L. Dunsch, S. Yang, H. S. Chakraborty,

M. E. Madjet and J. M. Rost 
Collision of Transition Metal Cluster Ions with Simple Molecules

M. Ichihashi, T. Hanmura and T. Kondow

\section{Part D. Clusters on a Surface}

Infrared Spectroscopy and Thermal Desorption Study of Vanadium-Mesitylene 1:2 Sandwich Clusters Soft-Landed onto a Long-Chain N-Alkanethiolate Self-Assembled

Monolayer

M. Mitsui, S. Doi, K. Ikemoto, S. Nagaoka

and A. Nakajima

Simulation of the Nanoindentation Procedure on Pure Nickel

on the Smallest Length Scale: A Simple Atomistic Level Model

205

P. Berke, M.-P. Delplancke-Ogletree, A. Lyalin,

V. V. Semenikhina and A. V. Solov'yov

\section{Part E. Phase Transitions, Fusion, Fission and Fragmentation in Finite Systems}

Quantum Structuring of ${ }^{4} \mathrm{He}$ Atoms Around Ionic Dopants: Energetics of $\mathrm{Li}^{+}, \mathrm{Na}^{+}$and $\mathrm{K}^{+}$from Stochastic Calculations

E. Coccia, E. Bodo, F. Marinetti, F. A. Gianturco, E. Yurtsever, M. Yurtsever and E. Yildirim

On the Theory of Phase Transitions in Polypeptides

A. V. Yakubovich, I. A. Solov'yov, A. V. Solov'yov and W. Greiner

Translational Kinetic Energy Released in the Dissociative Cascade of Charged Rare-Gas Clusters: Hints for Finite Size Phase Transitions?

F. Calvo and P. Parneix

Part F. Clusters in Laser Fields

Dynamics of Metal Clusters: Free, Embedded and Deposited

M. Bär, F. Fehrer, P.-G. Reinhard, P. M. Dinh,

E. Suraud, L. V. Moskaleva and N. Rösch 
Phase, Amplitude, and Polarization Shaping by

Interferometric Pulse Generation

A. Lindinger, S. M. Weber, F. Weise

and M. Plewicki

\section{Part G. Clustering Phenomenon in System of Various Degrees of Complexity}

Electron-Positron Clusters: Structure and Stability

V. K. Ivanov, R. G. Polozkov and A. V. Solov'yov

Spectroscopy of Neutral Retinal and GFP Chromophores in the Gas Phase

L. H. Andersen

The Energy Landscape as a Computational Tool

J. M. Carr and D. J. Wales

\section{Part H. Structure and Dynamics of Biomolecules}

Theoretical Framework for the Interpretation of NMR

Residual Dipolar Couplings of Unfolded Proteins

O. I. Obolensky, A. V. Solov'yov,

K. Schlepckow and H. Schwalbe

Computational Simulations of Antibody: Antigen Unbinding

E. S. Henriques and A. V. Solov'yov

Iron Mineral Based Magnetoreception Mechanism in Birds

Biophysical Modeling of Fragment Distributions of DNA

Plasmids After Heavy Ion Irradiation

Th. Elsässer, M. Scholz, G. Taucher-Scholz, S. Brons, K. Psonka and E. Gudowska-Nowak 
Towards Monte Carlo Calculations of Biological Dose in Heavy-Ion Therapy: Modeling of Nuclear Fragmentation Reactions

I. Pshenichnov, I. Mishustin and W. Greiner

Mechanisms of Radiation Damage of Biomolecules

E. Surdutovich, O. I. Obolensky, I. Pshenichnov, I. Mishustin, A. V. Solov'yov and W. Greiner

On Modeling of Gene Expression Patterns in the Drosophila Embryo by the Gene Circuit Method

A. M. Samsonov, V. V. Gursky, K. N. Kozlov and J. Reinitz

Author Index 